\section{ORIGINAL RESEARCH}

\author{
A. Bose \\ H. Henkes \\ K. Alfke \\ W. Reith \\ T.E. Mayer \\ A. Berlis \\ V. Branca \\ S. Po Sit, \\ for the Penumbra \\ Phase 1 Stroke Trial \\ Investigators
}

\title{
The Penumbra System: A Mechanical Device for the Treatment of Acute Stroke due to Thromboembolism
}

\begin{abstract}
BACKGROUND AND PURPOSE: Data from recent reports have indicated that mechanical thrombectomy may have potential as a treatment for acute ischemic stroke. The purpose of this study was to assess the safety and performance of the Penumbra System (PS): a novel mechanical device designed to reduce clot burden in acute stroke due to large-vessel occlusive disease.
\end{abstract}

\begin{abstract}
MATERIALS AND METHODS: A prospective, single arm, independently monitored and core laboratory adjudicated trial enrolled subjects with an acute neurologic deficit consistent with acute stroke, presenting within 8 hours of symptom onset and an angiographically verified occlusion (Thrombolysis in Myocardial Infarction [TIMI] grade 0 or 1) of a treatable intracranial vessel. The primary end point was revascularization of the target vessel to TIMI grade 2 or 3 . Secondary end points were the proportion of subjects who achieved a modified Rankin Scale (mRS) score of 2 or less or a 4-point improvement on the National Institutes of Health Stroke Scale (NIHSS) score at 30-day follow-up, as well as all-cause mortality.
\end{abstract}

\begin{abstract}
RESULTS: Twenty-three subjects were enrolled, and 21 target vessels were treated in 20 subjects by the PS. At baseline, mean age was 60 years, mean mRS score was 4.6, and mean NIHSS score was 21. Postprocedure, all 21 of the treated vessels $(100 \%)$ were successfully revascularized by the PS to TIMI 2 or 3. At 30-day follow-up, 9 subjects (45\%) had a 4-point or more NIHSS improvement or an $\mathrm{mRS}$ of 2 or less. The all-cause mortality rate was $45 \%$ (9 of 20), which is lower than expected in this severe stroke cohort, where $70 \%$ of the subjects at baseline had either an NIHSS score of more than 20 or a basilar occlusion.
\end{abstract}

CONCLUSION: Thus, early clinical experience suggests that the PS allows revascularization in certain subjects experiencing acute ischemic stroke.

C urrent thrombolytic therapy in acute ischemic stroke is often not effective or can be difficult to administer within the indicated brief treatment window of 3 hours from symptom onset. The average time from stroke onset to arrival in an emergency department is between 3 and 6 hours. Mortality rates associated with occlusions of the basilar artery, internal carotid artery (ICA) terminus, and M1 segment of the middle cerebral artery (MCA) are particularly high despite best available medical therapy. ${ }^{1-3}$ The paucity of safe and effective treatment strategies presents a profound unmet clinical need in the management of this prevalent and debilitating disease. Substantial evidence in the published literature suggests that early and safe revascularization of the primary occlusion correlates with improved clinical outcome. ${ }^{4}$ The probable basis for this correlation is that, although some brain tissue experiences near immediate and irreversible infarction during acute stroke, there remains a region of ischemic penumbra surrounding this area where at-risk tissue can be salvaged. ${ }^{4}$ Therefore, if timely revascularization can be effected and reperfusion established, then damage to the penumbral region could be reversed, resulting in a diminished neurologic deficit,

Received October 11, 2007; accepted after revision March 5, 2008.

From Lenox Hill Hospital (A.B.), New York, NY; Robert Janker Klinik (H.H.), Bonn, Germany; University of Kiel Medical Center (K.A.), Kiel, Germany; Universitätsklinikum des Saarlandes (W.R.), Homburg/Saar, Germany; Universität München Klinikum (T.E.M.), Munich, Germany; Universität Hospital (A.B.), Freiburg, Germany; Ospedale Maggiore Milano (V.B.), Milan, Italy; and Penumbra Inc (A.B., S.P.S.), San Leandro, Calif.

Please address correspondence to Siu Po Sit, Penumbra, Inc, 1351 Harbor Bay Pkwy, Alameda, CA 94502; e-mail: ssit@penumbrainc.com

DOI 10.3174/ajnr.A1110 reduced stroke-related mortality and morbidity, and improved clinical outcome.

With the advent of endovascular therapy, a number of alternative mechanical approaches have shown promise in restoring blood flow in an occluded artery in acute ischemic stroke., ${ }^{5,6}$ The Penumbra System (PS; Penumbra, Alameda, Calif) is a new embolectomy device specifically designed to remove the thrombus in acute ischemic stroke secondary to large vessel thromboembolism. The device removes the thrombus through 2 mechanisms: aspiration and extraction. The PS is composed of 3 main components: a reperfusion catheter, separator, and thrombus removal ring (Fig 1). For aspiration, the reperfusion catheter is used in parallel with the separator and an aspiration source to separate the thrombus and aspirate it from the occluded vessel. If residual thrombus remains after revascularization with aspiration, the thrombus removal ring is used to directly engage and remove the thrombus.

\section{Materials and Methods}

A prospective, multicenter, single-arm trial was designed to assess the safety and performance of the PS in acute ischemic stroke. The protocol called for up to 30 subjects with acute ischemic stroke to be enrolled at 6 international centers for treatment with the PS within 8 hours of symptom onset; however, only 23 subjects were enrolled due to a higher than expected revascularization rate (100\%). The primary end point was the ability of the PS to revascularize the affected vessel to Thrombolysis in Myocardial Infarction (TIMI) 2 or greater after use of the system. ${ }^{7}$ The secondary end points were the proportion of subjects who achieved a modified Rankin Scale (mRS) score of 2 or 

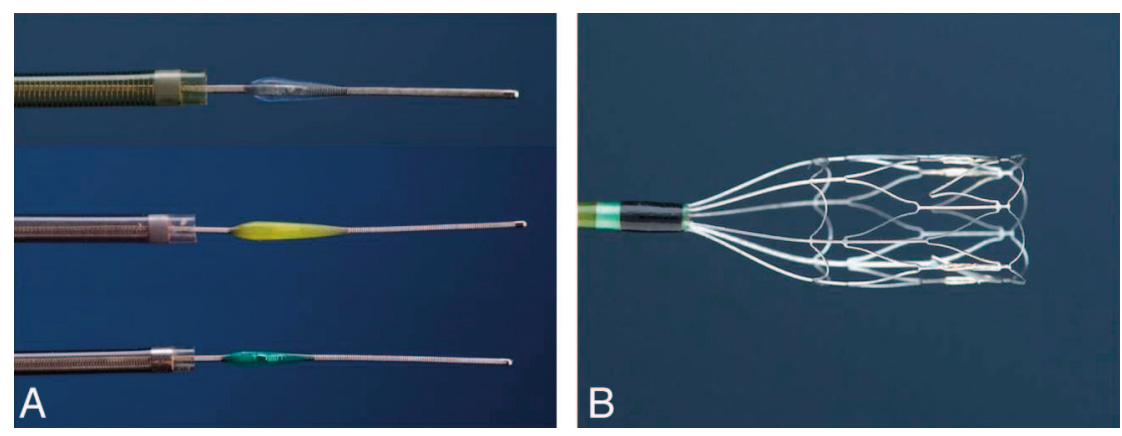

Fig 1. Schematic diagram of the Penumbra device.
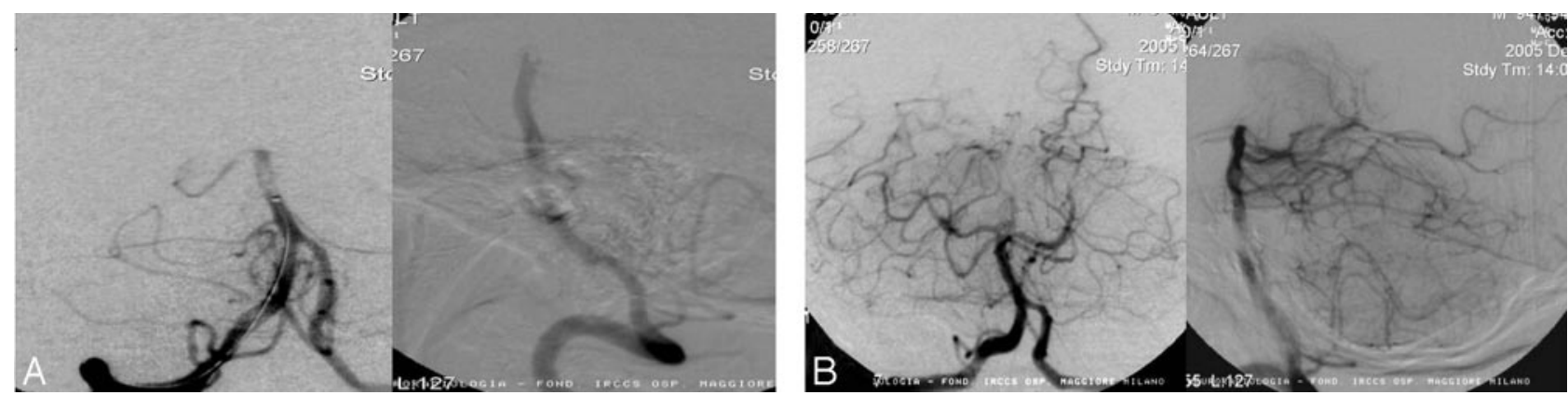

Fig 2. Angiographic illustration of a target vessel before $(A)$ and after $(B)$ treatment by the PS. This patient was a 66 -year-old man who presented 3 hours from symptom onset with an NIHSS score of 24, which improved to 8 at 24 hours after revascularization by the PS. He continued to recover with a 30-day NIHSS score of 2 and an mRS of 1.

less or a 4-point improvement on the National Institutes of Health Stroke Scale (NIHSS) at 30 days postprocedure. The 30-day all-cause mortality rate was also assessed.

\section{Inclusion and Exclusion Criteria}

The main entry criteria for the study were as follows: 1) clinical signs consistent with acute ischemic stroke; 2) age 18 years or older; 3 ) a score of TIMI 0 or 1 in a vessel accessible by the PS; and 4) presentation within 8 hours of stroke symptom onset (if presenting within 3 hours of stroke symptom onset, the subject must have been ineligible for or refractory to treatment with intravenous [IV] recombinant tissue plasminogen activation [rtPA]). Principal exclusion criteria for enrollment were as follows: subjects who were at risk of bleeding, vessels that were too tortuous for access by the PS, and subjects with severe, uncontrolled hypertension. Women who were pregnant were also excluded.

\section{Subject Selection}

Subjects presenting with stroke symptoms were screened to determine whether they met entry criteria for the study. After obtaining signed informed consent, all of the eligible subjects were evaluated for neurologic and functional status, and an angiographic assessment of the suspected vascular occlusion was obtained. An arterial access procedure was performed by using standard percutaneous techniques under systemic heparin and general anesthesia per the local institutional standard of care. For subjects without thrombolytic therapy onboard, heparin was given IV at a dose of 5000 IU bolus followed by a continuous infusion at $2000 \mathrm{IU} / \mathrm{h}$ to maintain an activated clotting time of 250 seconds or more. For subjects who had received thrombolytic therapy, it was given at a dose of 2000 IU bolus followed by a continuous infusion at $1000 \mathrm{IU} / \mathrm{h}$.

\section{Treatment Procedures}

Four-vessel digital subtraction angiography was used to define the angioarchitecture of the occluded vascular segment. An appropriate guide catheter was then brought into position in the occluded target vessel territory to enable access by the reperfusion catheter. All of the components of the PS are deliverable via a $6 \mathrm{~F}$ standard guide catheter. A subject was considered enrolled into the study when the reperfusion catheter was deployed from the guide catheter to the site of occlusion. Once the appropriate position was achieved proximal to the clot, the guidewire was removed from the reperfusion catheter, and the separator was advanced through the reperfusion catheter. The aspiration pump was then turned on to initiate revascularization. Reduction of the clot burden by aspiration was accomplished by connecting the reperfusion catheter to the aspiration pump, which generated a vacuum of -20 inches $\mathrm{Hg}$. A continuous aspiration-debulking process was facilitated by advancing and withdrawing the separator through the reperfusion catheter into the proximal end of the clot. If the thrombus remained, a second accessory method of direct mechanical retrieval by the thrombus removal ring was used to augment revascularization. Thrombus extraction by using the thrombus removal ring was accomplished by engaging the clot proximally and extracting the clot under flow arrest conditions by inflating a proximal balloon guide catheter. If the PS was successful in revascularization of the target vessel to TIMI 2 or better, no additional interventions were performed. Administration of anticoagulants and antiplatelets were suspended for 24 hours posttreatment. If the subject experienced symptoms that appeared to be indicative of an intracranial hemorrhage (ICH; neurologic deterioration or alteration in function), an emergent CT scan or MR imaging was conducted. A follow-up CT was required 14 days after the initial emergent CT.

\section{Study End Points}

The primary end point for this study was incidence of revascularization of the target vessel as defined by achieving TIMI 2 or 3 flow after use of the PS (Fig 2). ${ }^{7}$ Each investigator made an initial assessment of TIMI flow in the target vessel preprocedure and postprocedure. Preprocedure and postprocedure angiograms were sent to an independent core laboratory to make a final determination on TIMI flow. 


\begin{tabular}{|c|c|c|}
\hline \multicolumn{3}{|c|}{ TIMI scores (core laboratory adjudicated) } \\
\hline Variable & $\begin{array}{c}\text { Baseline } \\
(N=21), \%(n / M)\end{array}$ & $\begin{array}{c}\text { Posttreatment } \\
(N=21), \%(n / M)\end{array}$ \\
\hline TIMI 0 & $90(19 / 21)$ & $0(0 / 21)$ \\
\hline TIMI 1 & $10(2 / 21)$ & $0(0 / 21)$ \\
\hline TIMI 2 & $0(0 / 21)$ & $48(10 / 21)$ \\
\hline TIMI 3 & $0(0 / 21)$ & $52(11 / 21)$ \\
\hline
\end{tabular}

Note:-Twenty-one target vessels were treated in 20 subjects. TIMI indicates Thrombolysis in Myocardial Infarction.

Data based on core laboratory assessments were used for final analysis.

\section{Results}

Twenty-three subjects at 6 international centers were enrolled with 21 vessels treated by the PS. Three enrolled subjects were not treated due to vessel tortuosity, resulting in an access rate of $87 \%$ (20 of 23). Among the 20 subjects, 8 (40\%) were women. They had a mean age of $60 \pm 18( \pm$ SD), baseline mRS of $4.6 \pm 0.8$, and NIHSS score of $21 \pm 8$. Eleven $(45 \%)$ had a baseline NIHSS score of more than 20 . Fifty percent of the subjects (10 of 20) presented more than 3 hours from symptom onset. Of the remaining 50\% who presented within 3 hours, 6 were refractory to rtPA therapy, and 4 were not eligible for thrombolytic therapy. Among the 21 vessels treated by the PS, 7 (33\%) were ICA, 5 (24\%) were MCA, and $9(43 \%)$ were basilar arteries. Fourteen $(70 \%)$ of the subjects had either a baseline NIHSS score of more than 20 or a basilar occlusion. The 3 subjects who were enrolled but not treated were due to the inability of the PS to access the target lesions. Two of these subjects presented with left M1 occlusion and 1 with a site of primary occlusion at the right ICA supraclinoid region. There were no reports of adverse events during the procedures. All 3 of the subjects were discharged to either a rehab center or a nursing home. Two of the subjects survived through the 30day follow-up, of which one showed improvement in the NIHSS and the other remained stable. The third subject with the left M1 occlusion showed a small, asymptomatic ICH in the infarcted area from a follow-up CT at 3 days postprocedure. It did not progress, and her NIHSS score remained stable at 19 (from a baseline of 22). This subject died at 32 days postprocedure from worsening of pre-existing heart failure, unrelated to the procedure or the device. All of the treated target vessels were either TIMI 0 ( 19 of 21 ) or TIMI 1 ( 2 of 21) before treatment with the PS, and all were successfully revascularized to TIMI 2 or 3 after use of the Penumbra stroke system (21 of 21 ; Table 1 ).

In the study, additional interventions with intra-arterial (IA) rtPA were performed in 9 subjects after use of the PS, with doses ranging from 10 to $45 \mathrm{mg}$ given as a bolus. The main reason cited by the investigators for using IA rtPA was the intention to better the score from TIMI 2 due to evidence of vessel occlusion in the vasculature distal to the revascularized target vessel. The TIMI scores summarized above were recorded before any adjunctive treatment had been initiated, at a time when revascularization by the PS alone was considered complete. The secondary end points were the proportion of subjects who achieved an mRS of 2 or less or a 4-point improvement on the NIHSS at 30-day follow-up, as well as allcause mortality.

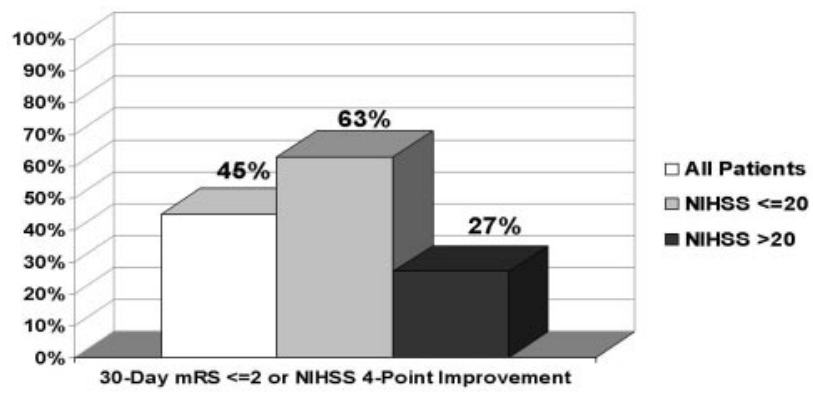

Fig 3. Subjects outcome based on baseline NIHSS scores.

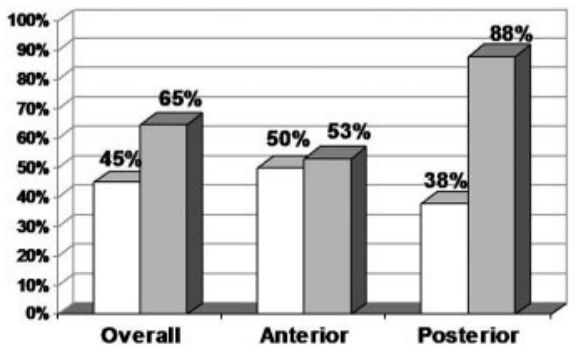

口Observed Mortality Rates 口Expected Mortality Rates

Fig 4. A comparison of the observed and expected mortality rates for the entire study cohort and after stratification by location of the target vessel. The expected rates were derived from historical rates from the natural history of the disease reported in the literature, prorated to the proportion of target vessel locations treated in this study (see References 1-3)

In the treated subjects, $45 \%$ (9 of 20) of subjects with 30 day data achieved at least a 4-point improvement of NIHSS or an mRS of 2 or less. A total of 9 subjects had an improvement of 4 or more points on the NIHSS score, and 7 subjects had an mRS of 2 or less. Results from a subgroup analysis indicate that subjects with a baseline NIHSS score of more than 20 tended to have lower probability of a good outcome at 30 days postprocedure (Fig 3).

Of the 20 enrolled subjects who were treated by the system, 9 died within the 30-day follow-up period, resulting in an all-cause mortality of $45 \%$ ( 9 of 20 ). This rate is lower than expected in this severe stroke cohort, where at baseline, $70 \%$ of the subjects had either an NIHSS score of more than 20 or a basilar occlusion (Fig 4). ${ }^{1-3}$ None of these deaths were related to the study device. The most frequently cited cause of death for these subjects was hemorrhagic conversion of a region of infarction and cerebral edema secondary to infarction.

Two procedural adverse events were reported from 21 procedures. One subject experienced a groin hematoma at the puncture site, which was treated with a transfusion and resolved. Another subject experienced an intraprocedural subarachnoid hemorrhage. The hemorrhage did not result in any deterioration of the subject's neurologic condition and resolved with no action taken. There were no other reports of arterial perforation, dissection, or embolization of a previously uninvolved territory of the brain.

There was a total of 8 reported cases of $\mathrm{ICH}$, of which 2 were symptomatic as defined by CT evidence of a bleeding event and a 4-point deterioration on the NIHSS score. ${ }^{8}$ In 7 of the 8 cases of ICH, IA thrombolytics were administered after use of the PS. In 1 of the 8 cases, IV thrombolytics were given before treatment with the PS. Among the 8 cases of hemorrhage, 1 was thought to have been caused by the device (see 
above description of intraprocedural subarachnoid hemorrhage). Adjunctive IA thrombolytic therapy was associated with a higher incidence of hemorrhage.

\section{Discussion}

Current medical practice offers physicians various treatment strategies in the management of acute ischemic stroke. Unfortunately, many treatment modalities face limitations that prevent substantive improvements of stroke-related morbidity and mortality.

The National Institute of Neurological Disorders and Stroke study found that subjects experienced the greatest benefit from treatment with rtPA if treatment was initiated within 3 hours of stroke symptom onset. ${ }^{9}$ At 3 months, rtPA-treated subjects were $30 \%$ more likely to have minimal or no disability compared with the placebo-treated subjects. Subjects in the treatment group did experience a higher rate of symptomatic $\mathrm{ICH}(6.4 \%$ versus $0.6 \%)$; however, the investigators showed the benefits of the treatment outweighed the risk. Because subjects are at a greater risk for hemorrhage with thrombolytic use after 3 hours of symptom onset, the study only enrolled those subjects who presented within 3 hours from symptom onset who had minimal bleeding-complication risk factors.

IV administration of thrombolytic agents requires a high dose to be given to the subject that may increase the risk of ICH. The Prolyse in Acute Cerebral Thromboembolism II Trial sought to assess whether local, IA administration of prourokinase at the site of the thrombus would be safe and effective for the treatment of ischemic stroke. ${ }^{10}$ Subjects presenting within 6 hours of stroke symptom onset and with occlusions of the MCA were enrolled, and subjects were randomly assigned to receive 9-mg IA recombinant prourokinase ( $r$-proUK) plus heparin or heparin alone (control). At 90 days, $40 \%$ of r-proUK-treated subjects and $25 \%$ of control subjects had an mRS of 2 or less. Mortality for the 2 groups was equivalent $(25 \%$ in the treatment arm versus $27 \%$ in the control arm), and the symptomatic ICH rate was $10 \%$ for the treatment group and $2 \%$ for the control group.

In an attempt to reduce systemic adverse effects associated with the use of thrombolytic agents, focus has shifted to mechanical rather than pharmaceutical means of recanalizing the site of primary occlusion in subjects experiencing acute ischemic stroke. Only one mechanical device, approved in a safety study, is available on the market in the United States and Europe for revascularization of occlusions caused by acute ischemic stroke (Merci Retriever; Concentric Medical, Mountain View, Calif). ${ }^{11-13}$ Published data for the Merci Retriever based on 141 treated subjects showed a revascularization rate (TIMI $2 / 3$ flow) of $48 \%$ with a clinically significant procedure-related adverse event rate of $7.1 \%$ (10 of 141). ${ }^{11}$ Eleven subjects (7.8\%) experienced a symptomatic ICH within 24 hours, and $27.7 \%$ had asymptomatic ICH. At 90 days, $43.5 \%$ of subjects died, and $27.7 \%$ of subjects had a good outcome ( $m R S \leq 2)$. In the Multi Mechanical Embolus Removal in Cerebral Ischemia Trial, where a combined therapy of the Merci Retriever and rtPA treatment (IV/IA) was assessed, the rate of revascularization was increased to $69.0 \%$, and mortality was reduced to $30.6 \%$ at 3 months. ${ }^{12}$ However, this was associated with an increase in symptomatic ICH to $9.0 \%$ and an increase in the asymptomatic ICH rate to $29.7 \%$. Similarly, in the Interven- tional Management of Stroke I Trial, a combined therapy of IV and IA rtPA resulted in a protocol-defined revascularization rate of $56 \%$, a symptomatic ICH rate of $6.3 \%$, and an asymptomatic ICH rate of $42.5 \%$.

By comparison, in this small cohort of subjects, the PS was able to revascularize the site of primary occlusion in all of the treated subjects enrolled into the study, resulting in a revascularization rate of $100 \%$, of which $45 \%$ had met the secondary end point of achieving a 4-point improvement on the NIHSS or an mRS of 2 or less at 30 days postprocedure. This was associated with a device or procedure-related serious adverse event rate of $10 \%$ and an all-cause mortality rate of $45 \%$.

Eight subjects were observed to have ICH in this study, of which $2(10 \%)$ were symptomatic and $6(30 \%)$ were asymptomatic. The risk of cerebral hemorrhage secondary to the use of IA rtPA therapy is controversial in the literature..$^{8,14-16}$ In this small cohort of patients, the observation of ICH was associated with the use of additional IA rtPA as adjunctive therapy to the PS to reduce the clot burden in distal branches beyond the site of primary occlusion. Additional studies may be warranted to better define the optimal use of adjunctive IA lytic therapy to reduce clot burden at the site of primary occlusion and to improve distal perfusion after the site of primary occlusion has been revascularized.

The device safety and revascularization success of the PS appears promising in this early clinical experience. In addition, the neurologic and functional improvement noted in some subjects is encouraging in this patient cohort where $70 \%$ of the subject at baseline had either an NIHSS score of more than 20 or a basilar occlusion. Further investigation seems warranted to better define the safety and efficacy of the PS in acute ischemic stroke.

\section{Conclusions}

In this prospective, phase 1, single arm trial, the PS allows revascularization of occluded intracranial large vessels in a small stroke cohort who presented within 8 hours from symptom onset. This early clinical experience suggests that the PS may have potential as treatment for acute ischemic stroke secondary to large-vessel thromboembolism and that further clinical testing is warranted.

\section{Clinical Investigators}

Participating centers principal investigator (PI) and coinvestigator(s) (Co) in order of enrollment $(n)$ : Robert Janker Klinik, Bonn, Germany: Hans Henkes, MD (PI), and Stefan Lowens, MD (Co) (6); University of Kiel Medical Center, Kiel, Germany: Prof Olav Jansen, MD (PI), and Karsten Alfke, MD (Co) (5); Universitätsklinikum des Saarlandes, Homburg/ Saar, Germany: Prof Wolfgang Reith, MD (PI), Prof Klaus Fassbender, MD (Co), Iris Grunwald, MD (Co), Joearg Osterhage, $\mathrm{MD}(\mathrm{Co})$, Panagiotis Papanagiotou, $\mathrm{MD}(\mathrm{Co})$, and Erdem Orberk, MD (Co) (5); Universität München Klinikum, Munich, Germany: Prof Thomas E. Mayer, MD (PI), Stephanie Muller-Schunk, MD (Co), Prof Martin Wiesmann, MD (Co), Prof Hartmut Bruckmann, MD (Co), Klaus Seelos, MD (Co), Gunther Fesl, MD (Co), Markus Holtmannspotter, MD (Co), Prof Martin Dichgans, MD (Co), and Yvonne Mewald, MD (Co) (3); Universität Hospital, Freiburg, Germany: Ansgar Berlis, MD (PI), Prof Martin Schumacher, MD (Co), 
Wolf-Dirk Niesen, MD (Co), and Andreas Hetzel, MD (Co) (2); and Ospedale Maggiore Milano, Milan, Italy: Vincenzo Branca, MD (PI) (2).

\section{Acknowledgments}

We thank Prof Dr Michael Knauth and the George-AugustUniversity, Bereich Humanmedizin, Göttingen, Germany, for their invaluable services as the angiographic core laboratory in this study.

\section{References}

1. Jansen $\mathrm{O}$, von Kummer R, Forsting M, et al. Thrombolytic therapy in acute occlusion of the intracranial internal carotid artery bifurcation. AJNR Am J Neuroradiol 1995; 16:1977-86

2. Brandt T, von Kummer R, Muller-Kuppers M, et al. Thrombolytic therapy of acute basilar artery occlusion. Stroke 1996;27:875-81

3. Eckert B, Koch C, Thomalla G, et al. Aggressive therapy with intravenous abcizimab and intra-arterial rtPA and additional PTA/stenting improves clinical outcome in acute vertebrobasilar occlusion: combined local fibrinolysis and intravenous abciximab in acute vertebrobasilar stroke treatment (FAST): results of a multicenter study. Stroke 2005;36:1160-65

4. Jansen O, Schellinger P, Fiebach J, et al. Early revascularization in acute ischemic stroke saves tissue at risk defined by MRI. Lancet 1997;353:2036-37

5. Leary M, Saver S, Gobin Y, et al. Beyond tissue plasminogen activator: mechanical intervention in acute stroke. Ann Emerg Med 2003;41:838 -46
6. Gomez CR, Orr SC, Soto RD. Neuroendovascular rescue: Interventional treatment of acute ischemic stroke. Curr Treat Options Cardiovasc Med 2002;4:405-19

7. Thrombolysis in Myocardial Infarction (TIMI) Trial: phase 1 findings - TIMI Study Group. N Engl J Med 1985;312:932-36

8. Khatri P, Wechsler LR, Broderick JP. Intracranial hemorrhage associated with revascularization therapies. Stroke 2007;38:431-40

9. The National Institute of Neurological Disorders and Stroke t-PA Stroke Study Group. Tissue plasminogen activator for acute ischemic stroke. N Engl J Med 1995;333:1581-87

10. Furlan A, Higashida R, Wechsler L, et al. Intra-arterial prourokinase for acute ischemic stroke. The PROACT II Study: a randomized controlled trial. JAMA 1999;282:2003-11

11. Smith WS, Sung G, Starkman S, et al. Safety and efficacy of mechanical embolectomy in acute ischemic stroke: results of the MERCI trial. Stroke 2005;36:1432-38

12. Smith WS. Safety of mechanical thrombectomy and intravenous tissue plasminogen activator in acute ischemic stroke. Results of the multi Mechanical Embolus Removal in Cerebral Ischemia (MERCI) trial, part I. AJNR Am J Neuroradiol 2006;27:1177-82

13. Gobin YP, Starkman S, Duckwiler GR, et al. MERCI 1: a phase 1 study of mechanical embolus removal in cerebral ischemia. Stroke 2004;35:2848-54

14. Ogawa A, Mori E, Minematsu K, et al. Randomized trial of intraarterial infusion of urokinase within 6 hours of middle cerebral artery stroke. The middle cerebral artery embolism local fibrinolytic intervention trial (MELT) Japan. Stroke 2007;38:2633-39

15. Saver JL. Intra-arterial fibrinolysis for acute ischemic stroke. The message of MELT. Stroke 2007;38:2627-28

16. IMS Trial Investigators. Combined intravenous and intra-arterial revascularization for acute ischemic stroke: the interventional management of stroke study. Stroke 2004;35:904-11 